https://doi.org/10.15407/ujpe64.7.619

V. PUGATCH

on behalf of the $\mathrm{LHCb}$ Collaboration

Institute for Nuclear Research, Nat. Acad. of Sci. of Ukraine

(47, Prosp. Nauky, Kyiv 03680, Ukraine; e-mail: pugatch@kinr.kiev.ua)

\title{
HEAVY-ION AND FIXED-TARGET PHYSICS IN LHCb
}

\begin{abstract}
Selected results of the $\mathrm{LHCb}$ experiment on heavy ion collisions studied in the collider and fixed-target modes are presented. The clear evidence of the impact of the production mechanism (prompt/delayed, $p$ - $p$ or $p$-Pb systems) on the $\mathrm{p}_{T}$ and rapidity distributions for $J / \psi, D^{0}$ and $\Upsilon(n s)$ species is demonstrated. The interpretation of the observations in frames of theoretical models is briefly discussed. Some original results, as well as prospects of fixed-target mode studies, are presented.
\end{abstract}

Keywords: high-ehergy physics, heavy ions, LHCb experiment, nuclear modification factor, quark-gluon plasma.

\section{Introduction}

The LHCb Collaboration has started heavy ion studies in the year 2013, and many interesting observations have been reported. In this presentation, we shall discuss recent results on the charmonium and bottonium production cross-sections measured over the transverse momentum and rapidity. Physics goals include studies of the hadronic matter at high densities and temperatures, nucleon and nuclear PDFs, dynamics of the multinucleon interaction, hadronization, and QED at high electromagnetic field strengths. Charmonium and bottomonium states are considered as tools for the studies. It is assumed that their features are dependent on the properties of the QGP. One can expect their dependence on the interaction energy (collider or fixed-target mode), systems size (p-p, p-A, A-A), localization of the quarkonium production and direction of its emission (primary interaction region or displaced vertices, forward or backward emission), and different levels of a modification for the ground and excited states of the same probe, as well as on the centrality factor or multiplicity of events. To quantify the above-mentioned impacts, it is natural to compare differential production cross-sections measured in the proton proton scattering and in heavy ion collisions (p-A, A-A) at the same nucleon-nucleon cms energy. The normalized ratio of those cross-sections is defined as a Nuclear Modification Factor (NMF). The LHCb experiment operating in the collider and fixed-target modes allows one

(C) V. PUGATCH, 2019

ISSN 2071-0194. Ukr. J. Phys. 2019. Vol. 64, No. 7 to measure the double differential production crosssections for various hadronic probes as a function of the transverse momentum and the rapidity. The measured physical observables treated theoretically allow one to probe the structure of nuclei on the partonic scale. In this presentation, selected recent results on heavy ion collisions in the collider and fixed-target modes are presented for $J / \psi, D^{0}$, and $\Upsilon(n s)$ hadrons.

\section{2. $\mathrm{LHCb}$ Detector}

The LHCb detector [1] is a forward spectrometer with excellent characterisitics: accep $_{T}$ ance $2<\eta<5$ (with HERSCHEL $8<\eta<10$ ), momentum resolution about $0.5 \%$, track reconstruction efficiency $>96 \%$, impact parameter resolution $\sim 20 \mu \mathrm{m}$ (decay time resolution: $\sim 45 \mathrm{fs}$ ), invariant mass resolution $\sim 15 \mathrm{MeV} / c^{2}$, and perfect particle identification efficiency in Ring-Imaging Cherenkov Detectors and the Muon system. LHCb is the only experiment at the LHC fully instrumented for the largerapidity range. The proton-lead collisions were studied at two energies corresponding to the protonnucleon center-of-mass energies $\sqrt{S_{N N}}=5.02 \mathrm{TeV}$ and 8.16 TeV. Protons and lead ions at fixed targets $(\mathrm{Ar}, \mathrm{He}, \mathrm{Ne})$ were studied at energies $\sqrt{S_{N N}}$ of $\sim 0.1 \mathrm{TeV}$. The directions of proton and lead beams were swapped during the data-taking period. The configuration with the protons traveling in the direction from the Vertex detector (VELO) to the Muon system is referred to as $\mathrm{p}-\mathrm{Pb}$ collisions, the inverse configuration as $\mathrm{Pb}-\mathrm{p}$ ones. The positive rapidity in the proton-nucleon center-of-mass system is defined

619 


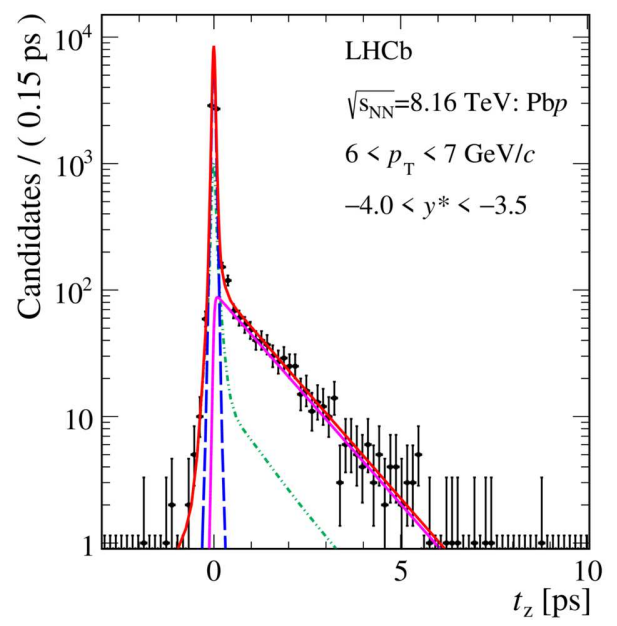

Fig. 1. Decay time distribution for $J / \psi$ events from $\mathrm{Pb}-\mathrm{p}$ collisions

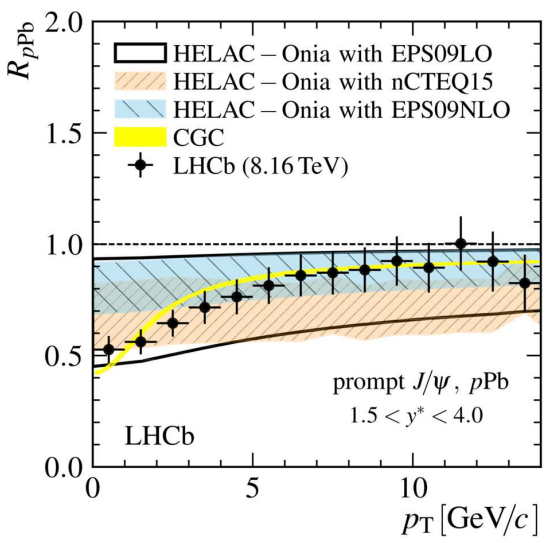

Fig. 2. NMF ( experiment - black circles with error bars) as a function of $\mathrm{p}_{T}$ for prompt $J / \psi$ in the rapidity range $1.5<y^{*}<4.0$

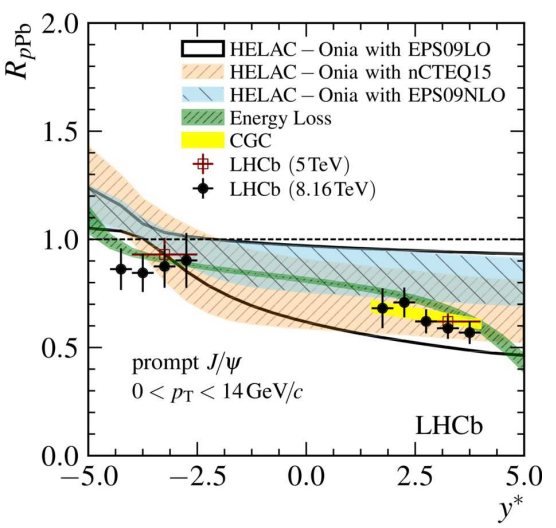

Fig. 3. Nuclear modification factor as a function of the rapidity for $J / \psi$ from prompt events by the direction of the proton beam. Thus, within a single experiment, the production cross-sections, forward-backward asymmetries, and nuclear modification factors were measured in a wide energy range for various hadronic states $\left(J / \psi, D^{0}, \Upsilon(n s)\right.$, antiprotons, $B$ mesons, and other hadrons).

\section{Charmonium Production in Proton-Lead Collisions at $\sqrt{S_{N N}}=5.02 \mathrm{TeV}$ and $8.16 \mathrm{TeV}$}

Exploring the powerful vertexing tool of the detector, the double differential cross-sections were measured for prompt and delayed $J / \psi$ mesons separated as illustrated in Fig. 1 [2]. The two components in the $J / \psi$ decay time distribution (Fig. 1) correspond to the prompt $J / \psi$ (narrow peak at zero time) and non-prompt $J / \psi$ from the b-hadron falling exponentially with a time constant of beauty hadrons. The interpretation of the results in frames of different theoretical approaches is illustrated in Fig. 2, where the experimental values for the NMF ( black circles with error bars) are shown as a function of $\mathrm{p}_{T}$ for prompt $J / \psi$ in the rapidity range $1.5<y^{*}<4$.0. If there would be no impact of the media, the NMF at the level of unity should follow up the dotted line parallel to the $x$-axis. Instead, a strong suppression of the $J / \psi$ production is well pronounced for $\mathrm{p}_{T}$ less than $10 \mathrm{GeV} / \mathrm{c}$. The CGC theory [3] follows experimental points nearly ideally, while other calculations (HELAC) just reflect the general tendency with large uncertainties [2]. These results constrain nPDFs in unexplored area at low- $x[4,5]$. The comparison of $\mathrm{p}_{T}$, as well as rapidity distributions, has revealed significant differences for $J / \psi$ mesons produced in $\mathrm{p}-\mathrm{p}$ and $\mathrm{p}-\mathrm{Pb}$ collisions. The data extracted from $\mathrm{p}-\mathrm{Pb}$ collisions at $8.16 \mathrm{TeV}$ for $J / \psi$ originated from primary vertices (prompt events, forward rapidity region) demonstrated a reduction of the cross-section by twice for low $\mathrm{p}_{T}(<4 \mathrm{GeV} / \mathrm{c})$. While, for the backward rapidity range, the cross-sections are close to be equal within statistical errors. The delayed events are characterized by a much less suppression even for the forward rapidity range $y^{*}$. These observations are consistent with data measured for $J / \psi$ at a lower energy of $5 \mathrm{TeV}$. This is illustrated in Figs. 3 and 4 [2] which show the nuclear modification factor extracted from data measured at $5 \mathrm{TeV}$ (open circles) and $8.16 \mathrm{TeV}$ (filled circles) for $J / \psi$ from prompt (Fig. 3) and from the decay of $b$-hadrons (Fig. 4). The remarkable dependence on the mechanism of produc- 
tion is clearly visible. The theoretical approach based on NLO nuclear PDFs accounting for the coherent energy-loss (black thick line) [6] follows well experimental data points for prompt $J / \psi$ (Fig. 3). Nonprompt $J / \psi$ are treated less satisfactorily with large uncertainties in frames of the calculations within the code FONLL with EPS09NLO [7] (Fig. 4). The production suppression at the forward rapidity for $J / \psi$ from $b$-hadrons is less pronounced than for prompt $J / \psi$. These data allow one to constrain nPDFs at low- $x$ [7]. Studies of the prompt $D^{0}$ meson production in $\mathrm{pPb}$ collisions at $5 \mathrm{TeV}[8]$ have demonstrated similar observations. As an example, Figs. 5 and 6 show data for the prompt $D^{0}$ meson production in $\mathrm{p}-\mathrm{Pb}$ collisions as a function of $y^{*}$ (Fig. 5) and the Nuclear modification factor $R_{\mathrm{p}-\mathrm{Pb}}$ as a function of $y^{*}$ (Fig. 6) with $\mathrm{p}_{T}<10 \mathrm{GeV} / \mathrm{c}$. The strong suppression in the forward-rapidity range (Fig. 6) was observed and well approximated by the theoretical description based on Nuclear PDFs and Color Glass Condensate assumptions. The data allow one to constrain nPDFs at low-Bjorken $x[7]$.

\section{Bottonium Production in Proton-Lead Collisions at 8.16 TeV}

Comprehencive studies were performed for properties of bottonium states $\Upsilon(1 S), \Upsilon(2 S)$, and $\Upsilon(3 S)$ produccd in $\mathrm{p}-\mathrm{p}$ and $\mathrm{p}-\mathrm{Pb}$ collisions [9]. The detailed analysis of $\mathrm{p}_{T}$, as well as rapidity distributions, demonstrates the suppression of $1 \mathrm{~S}$ and $2 \mathrm{~S}$ states, with the $2 \mathrm{~S}$ state being suppressed to a larger extent. Figures 7 and 8 show an example of such analysis for the nuclear modification factors $R_{\mathrm{p}-\mathrm{Pb}}$ for the $\Upsilon(1 S)$ and $\Upsilon(2 S)$ states (black dots with error bars) compared with different theoretical calculations (bands). $R_{\mathrm{p}-\mathrm{Pb}}(1 \mathrm{~S})$ is consistent with unity at negative $y^{*}$, while a significant (by $\sim 30 \%$ ) suppression is observed for positive $y^{*}$. The nuclear modification factor for $\Upsilon(2 S)$ is smaller than $\Upsilon(1 S)$ ) especially in the backward region, which is consistent with the comovers models [10] and in agreement with other experiments [11]. Calculations are based on the comovers model of $\Upsilon(n S)$ production, which implements the final state interaction of the quarkonia states and a nuclear parton distribution function modification. For the $\Upsilon(1 S)$ state, the nuclear modification factor is consistent with unity for $\mathrm{p}_{T}>10$ $\mathrm{GeV} / \mathrm{c}$, as predicted by the models. It is important to point out that the measurements of $B^{+}, B^{0}$, and $\Lambda^{0}{ }_{b}$

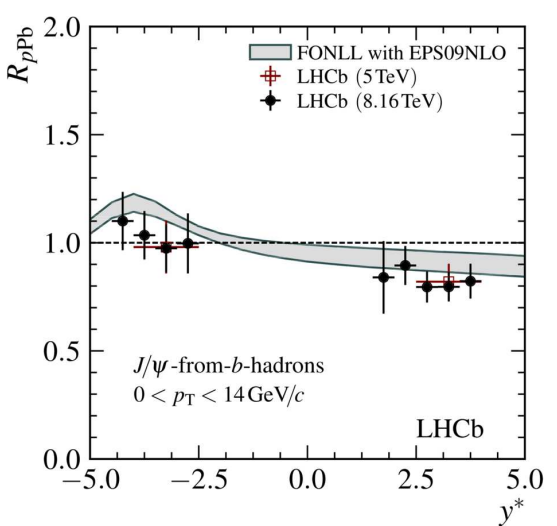

Fig. 4. Nuclear modification factor as a function of the rapidity for $J / \psi$ from $b$-hadrons

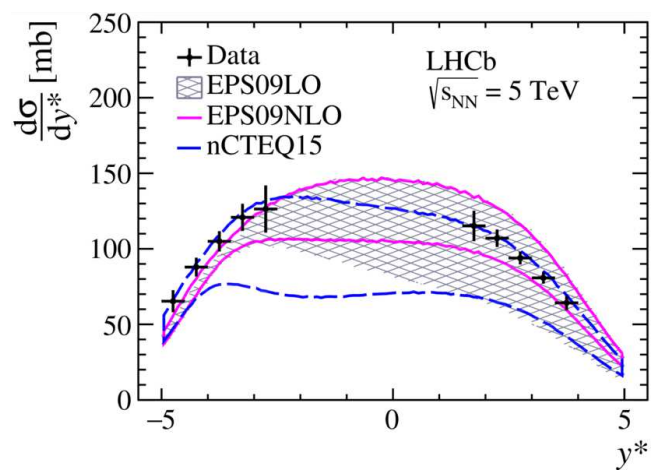

Fig. 5. Differential cross-section of the prompt $D^{0}$ meson production in $\mathrm{p}-\mathrm{Pb}$ collisions as a function of $y^{*}$

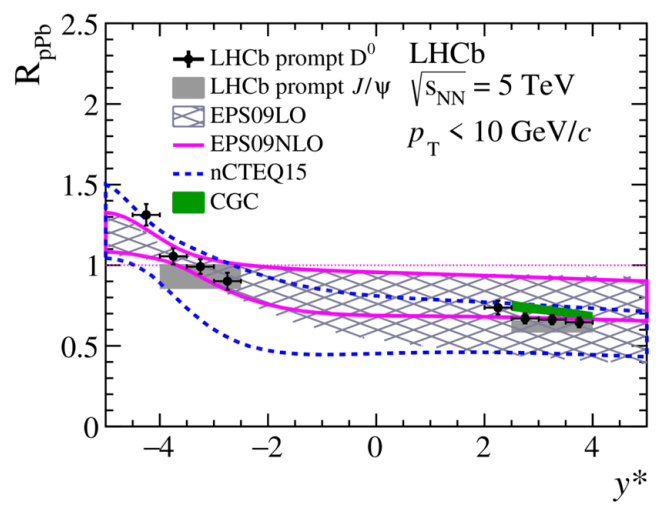

Fig. 6. Nuclear modification factor $R_{\mathrm{p}-\mathrm{Pb}}$ as a function of $y^{*}$ for the prompt $D^{0}$ meson production with $\mathrm{p}_{T}<10 \mathrm{GeV} / \mathrm{c}$

production in $\mathrm{pPb}$ collisions at $8.16 \mathrm{TeV}$ [12] also indicated a significant nuclear suppression of the nuclear modification factors and forward-to-backward crosssection ratios at the positive rapidity. 


\section{Fixed-Target Mode Studies}

The LHCb fixed-target program [13] includes, in particular, the study of the heavy-quark production in the large Bjorken $x$ region, the test of the intrinsic charm content of the proton and cosmic rays physics relevant production of antiprotons. Below, some results obtained in this area are briefly presented. In particular, the antiproton production in proton-helium collisions and the charmonium production in proton-argon collisions are discussed. The prompt anti-p production in $\mathrm{p}-\mathrm{He}$ collisions was thourouphly studied in a fixed-target mode at the energy $\sqrt{S_{N N}}=110 \mathrm{GeV}$ exploring a He gas injected inside by the SMOG system [14]. These data are important for the interpretation of recent results on the antiproton fraction in cosmic rays. An increase of the antiproton fraction in cosmic rays might be a sign of the antimatter produced by the dark matter annihilation. The measured production cross-sections were interpreted in frames of the several models differing by hadronization, parton model, and dynam-

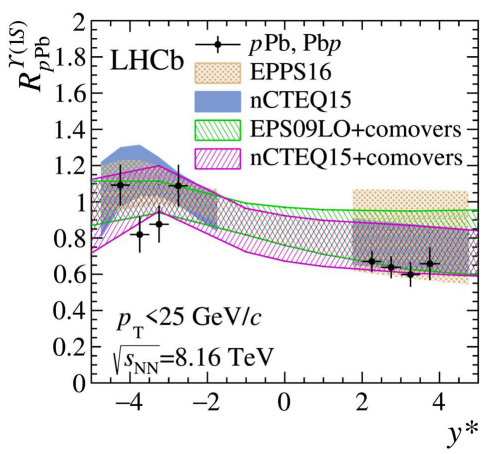

Fig. 7. Nuclear modification factor $R_{\mathrm{p}-\mathrm{Pb}}$ as a function of the rapidity $y^{*}$ for $\Upsilon(1 S)$ state

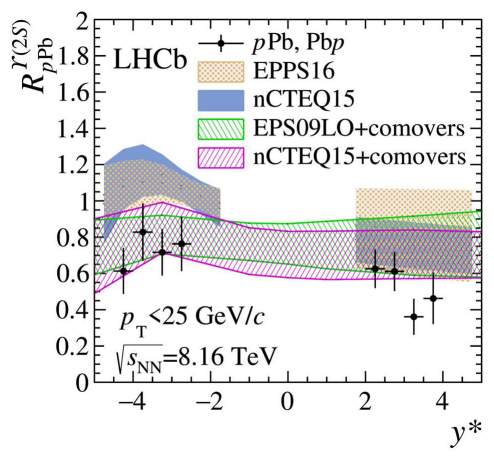

Fig. 8. Nuclear modification factor $R_{\mathrm{p}-\mathrm{Pb}}$ as a function of the rapidity $y^{*}$ for $\Upsilon(2 S)$ state ics. The shapes are well reproduced except at low rapidities, and the absolute yields deviate up to a factor of two [15]. The uncertainties $(\sim 10 \%)$ of experimental data are smaller than the spread of theoretical models. The results contribute to shrink background uncertainties in the dark matter searches in space $[15,16]$. Among other important resuts obtained in a fixed-target mode exploring the SMOG system, the first measurement of the heavy flavor $\left(J / \psi\right.$ and $\left.D^{0}\right)$ production cross-section in p-He at $\sqrt{S_{N N}}=86.6$ $\mathrm{GeV}$ and p-Ar at $\sqrt{S_{N N}}=110 \mathrm{GeV}$ at the LHC were reported in [18]. The measurements were performed in the range of $J / \psi$ and $D^{0}$ transverse momentum $\mathrm{p}_{T}<8 \mathrm{GeV} / \mathrm{c}$ and the rapidity $2.0<y<4.6$. In this range, any substantial intrinsic charm contribution should be seen in the p-He results. The measurements show no strong differences between p-He data and the theoretical predictions which do not consider the intrinsic charm contribution. Future measurements with larger samples and more accurate theoretical predictions will permit one to perform more quantitative studies.

In view of the successful running in the fixed-target mode in Run2, it is decided to upgrade the system for the injection of a gas for Run3. The SMOG2 [19] will inject a gas inside a $20-\mathrm{cm}$-long storage cell $(1 \mathrm{~cm}$ in diameter) in front of the vertex detector aiming to provide the instantaneous luminosity higher by up to two orders of magnitude. In addition to the noble gases, hydrogen and deuterium will operate as well. To extend the Heavy Ion Fixed Target program for Run4 and further, a crystal target, polarized target, and superthin wire targets were proposed and discussed. The LHCb fixed-target mode is unique for the experiments at $\mathrm{LHC}$, and it is planned to extend this area of studies in the future RUN3 and Run4 data taking.

\section{Summary and Outlook}

Double differential cross-sections for the production of charm and beauty hadrons measured in the collider and fixed-target mode in various combinations of heavy ions collisions at 5, 8, and $0.1 \mathrm{TeV}$ have been presented. The remarkable feature was observed in the collider-mode data: significant suppression of cross-sections at low transverse momenta and the forward rapidity in comparison with p-p data. The interpretation of the obtained results has been performed in frames of several theoretical approaches. The sta- 
tistical and theoretical uncertainties have to be reduced for improving the extraction of nPDFs.

We express our gratitude to our colleagues in the CERN accelerator departments for the excellent performance of the $\mathrm{LHC}$. We thank the technical and administrative staff at the LHCb institutes. We acknowledge the support from CERN and from the national agencies: CAPES, CNPq, FAPERJ and FINEP (Brazil); MOST and NSFC (China); CNRS/IN2P3 (France); BMBF, DFG and MPG (Germany); INFN (Italy); NWO (Netherlands); $M N i S W$ and NCN (Poland); MEN/IFA (Romania); MSHE (Russia); MinECo (Spain); SNSF and SER (Switzerland); NASU (Ukraine); STFC (United Kingdom); NSF (USA). We acknowledge the computing resources that are provided by CERN, IN2P3 (France), KIT and DESY (Germany), INFN (Italy), SURF (Netherlands), PIC (Spain), GridPP (United Kingdom), RRCKI and Yandex LLC (Russia), CSCS (Switzerland), IFIN-HH (Romania), CBPF (Brazil), PL-GRID (Poland) and OSC (USA). We are indebted to the communities behind the multiple opensource software packages on which we depend. Individual groups or members have received the support from AvH Foundation (Germany); EPLANET, Marie Sklodowska-Curie Actions and ERC (European Union); ANR, Labex P2IO and OCEVU, and Région Auvergne-Rhône-Alpes (France); Key Research Program of Frontier Sciences of CAS, CAS PIFI, and the Thousand Talents Program (China); RFBR, RSF, and Yandex LLC (Russia); GVA, XuntaGal, and GENCAT (Spain); the Royal Society and the Leverhulme Trust (United Kingdom); Laboratory Directed Research and Development program of $L A N L$ (USA). The studies have been partially supported in frames of the Targeted research program (Fundamental research on high-energy physics and nuclear physics, international cooperation) of the NAS of Ukraine and LIA IDEATE (STCU Project P9903).

1. LHCb Collaboration. A.A. Alves Jr. et al. The LHCb detector at the LHC. JINST 3, S08005 (2008).

2. LHCb Collaboration. R. Aaij et al. Prompt and nonprompt $J / \psi$ production and nuclear modification in $\mathrm{pPb}$ collisions at $\sqrt{s_{N N}}=8.16 \mathrm{TeV}$. Phys. Lett. B 774, 159 (2017).

3. B. Ducloué, T. Lappi, H. Mäntysaari. Forward $J / \psi$ production in proton-nucleus collisions at high energy. Phys. Rev. D 91, 114005 (2015).

4. J.-P. Lansberg, H.-S. Shao. Towards an automated tool to evaluate the impact of the nuclear modification of the gluon density on quarkonium, $D$ and $B$ meson production in proton-nucleus collisions. Eur. Phys. J. C 77, 1 (2017).

5. A. Kusina, J.-P. Lansberg, I. Schienbein, H.-S. Shao. Gluon shadowing in heavy-flavor production at the LHC. Phys. Rev. Lett. 121, 052004 (2018).

6. F. Arleo, S. Peigné. Heavy-quarkonium suppression in pA collisions from parton energy loss in cold QCD matter. JHEP 03, 122 (2013).

7. M. Cacciari, M. Greco, P. Nason. The $p_{T}$ spectrum in heavy-flavour hadroproduction. JHEP 05, 007 (1998).

8. LHCb collaboration, R. Aaij et al. Study of prompt $D^{0}$ meson production in $\mathrm{pPb}$ collisions at $\sqrt{s_{N N}}=5 \mathrm{TeV}$. JHEP 10, 090 (2017).

9. LHCb collaboration, R. Aaij et al. Study of $\Upsilon$ production in $\mathrm{pPb}$ collisions at $\sqrt{s_{N N}}=8.16 \mathrm{TeV}$. JHEP 11, 194 (2018).

10. E.G. Ferreiro, J.-P. Lansberg. Is bottomonium suppression in proton-nucleus and nucleus-nucleus collisions at LHC energies due to the same effects? JHEP 10, 94 (2018).

11. ATLAS Collaboration, M. Aaboud et al. Measurement of quarkonium production in proton-lead and proton-proton collisions at $5.02 \mathrm{TeV}$ with the ATLAS detector. Eur. Phys. J. C 78, 171 (2018).

12. LHCb collaboration, R. Aaij et al. Measurement of $B^{+}, B^{0}$ and $\Lambda_{\bar{b}}^{0}$ production in pPb collisions at $\sqrt{s_{N N}}=8.16 \mathrm{TeV}$. Phys. Rev. D 99, 052011 (2019).

13. S.J. Brodsky et al. Physics opportunities of a fixed-target experiment using LHC beams. Phys. Rep. 522, 239 (2013).

14. LHCb collaboration, R. Aaij et al. Precision luminosity measurements at LHCb. JINST 9, P12005 (2014).

15. LHCb collaboration, R. Aaij et al. Measurement of antiproton production in $p-\mathrm{He}$ collisions at $\sqrt{s_{N N}}=110 \mathrm{GeV}$. Phys. Rev. Lett. 121, 222001 (2018).

16. M. Korsmeier, F. Donato, Ma. Di Mauro. Production cross sections of cosmic antiprotons in the light of new data from the NA61 and LHCb experiments. Phys. Rev. D 97, 103019 (2018).

17. M. Boudaud et al. AMS-02 antiprotons are consistent with a secondary astrophysical origin. arXiv:1906.07119 (2019).

18. LHCb collaboration, R. Aaij et al. First measurement of charm production in its fixed-target configuration at the LHC. Phys. Rev. Lett. 122, 132002 (2019).

19. LHCb collaboration, I. Bediaga et al. LHCb SMOG Upgrade. CERN/LHCC 2019-005. LHCb TDR 2008 May 2019.

В. Пугач, від імені Колаборацї $L H C b$

Received 08.07.19

ФІЗИКА ВАЖКИХ ІОНІВ

ТА ФІКСОВАНОÏ МІШЕНI В ЕКСПЕРИМЕНТI LHCb

$\mathrm{P}$ е $з$ ю м е

Представлено вибрані результати експерименту LHCb по зіткненням важких іонів, дослідженим в колайдерному режимі та з фіксованою мішенню. Спостережено незаперечний вплив механізму (миттєвого чи з затримкою, в р-p чи $\mathrm{p}-\mathrm{Pb}$ системах) утворення мезонів $J / \psi, D^{0}$ або $\Upsilon(n s)$ на розподіли подій по $\mathrm{p}_{T}$ та бистротам. Коротко обговорюється інтерпретація спостережень у рамках теоретичних моделей. Представлено деякі оригінальні результати, а також перспективи досліджень в режимі фіксованої мішені. 